\title{
Estratégias de gerenciamento de riscos psicossociais no trabalho das equipes de saúde da família ${ }^{1}$
}

\section{Strategies of the management of the psychosocial risks in the work of the teams' family health}

\section{Estratégias de gerencia de los riesgos psicosociales en el trabajo de los equipos de salud de la familia}

\author{
Silvia Helena Henriques Camelo', Emília Luigi Saporiti Angerami"
}

\section{RESUMO}

O Programa de Saúde da Família tem o objetivo de reorganizar a atenção básica à saúde através de equipes multiprofissionais que correspondam às necessidades da população. Desenvolver este tipo de atividade junto à comunidade, onde a realidade do indivíduo é próxima, os problemas são de diversas ordens e as limitações incontáveis, expõem os trabalhadores a riscos psicossociais, tornando-o pessoa vulnerável ao estresse. Esta investigação tem o objetivo de identificar e analisar as estratégias utilizadas pelos trabalhadores para gerenciar os riscos psicossociais presentes no ambiente de trabalho. Este estudo do tipo exploratório utilizou-se da abordagem qualitativa, através do método de análise de conteúdo, modalidade temática. Participaram do estudo 24 trabalhadores pertencentes a seis equipes de saúde da família e os dados foram coletados por meio de uma entrevista que transcorreu no ano de 2005. As estratégias utilizadas pelos trabalhadores para o controle dos riscos são predominantemente individuais, tais como: exercício físico, religião e cinema. No entanto, o serviço tem uma reunião semanal programada com a equipe, para discussão dos problemas e sugestões. É inquestionável a importância de intervenções que promovam resultados benéficos ao trabalhador a nível psicológico e fisiológico, minimizando assim os efeitos dos eventos geradores de estresse no trabalho.

Palavras chave: Gerenciamento de riscos; Riscos ocupacionais; Equipe de assistência ao paciente; Saúde da Família.

\section{ABSTRACT}

The Program of Health Family has the objective of the reorganization the basic health attention through of professional teams that fulfill the necessity of the population. The development of this activity besides the community, where the reality of individual is near, the problems are diverse and the limitations are present, expose the workers to the physical and psychosocial risks that can to cause stress. This investigation identified and analyzed the control strategies utilized to alleviate fraying effects of those risks. This study had a character mainly exploratory and the analysis of the present study was based on the qualitative interpretation, thematic modality. The participants in this study were 24 workers and was employed a interview that it happened in the year of 2005. The strategies utilized to the relieve of the risks are mainly individuals such as physical exercise, religion and cinema, however, the institution contribute organizing meetings weekly, to discuss problems and create suggests. The interventions that promote benefic results to the worker in terms of psychological and physiologic can relieve the effects of the stress situations at work.

Key words: Safety management; Occupational risks; Patient Care team; Family Health.

\section{RESUMEN}

El Programa de Salud de la Familia (tiene como objetivo reorganizar la atención básica de la salud a través de equipos multiprofesional, que correspondan a las necesidades de la población. Desarrollar este tipo de actividades junto a la comunidad, donde la realidad del individuo es próxima, los problemas son de diversas órdenes y se enfrentan innúmeras limitaciones que exponen a los trabajadores a riesgos psicosociales, tornándolos personas vulnerables al estrés Esta investigación tiene como objetivo

\footnotetext{
Trabalho extraído da Tese de Doutorado apresentada à Escola de enfermagem de Ribeirão Preto- USP.

Profa. Drá. do Centro Universitário Barão de Mauá de Ribeirão Preto-SP. E-mail: jscamelo@uol.com.br.

"Profa. Titular aposentada da Escola de Enfermagem de Ribeirão Preto-USP.
} 
identificar y analizar las estratégias utilizadas por los trabajadores para enfrentar los riesgos presentes en el ambiente de trabajo. En este estudio de tipo exploratorio se utilizó abordaje cualitativo, a través del Método de Análisis de Contenido, modalidad Temática. Participaron del estudio veinticuatro (24) trabajadores pertenecientes a seis equipos de salud de la familia y los datos fueron recolectados por medio de una entrevista que sucedió en el ano de 2005. Las estratégias utilizadas por los trabajadores para el control de los riesgos son predominantemente individuales, tales como:

\section{NTRODUÇÃO}

Nas últimas décadas, a crise estrutural do setor público gera um hiato entre os direitos constitucionalmente garantidos e a efetiva capacidade de oferta dos serviços públicos associados aos mesmos, sendo percebida na fragilidade apresentada tanto na eficiência como na eficácia da gestão das políticas sociais e econômicas.

O Programa de Saúde da Família (PSF) desponta como uma das estratégias assumidas pelo Ministério da Saúde e tem o objetivo geral de "melhorar o estado de saúde da população mediante a construção de um modelo assistencial baseado na promoção, proteção, diagnóstico precoce, tratamento e recuperação da saúde", em conformidade com os princípios e diretrizes do SUS ${ }^{(1)}$.

Um desafio central do programa é mostrar sua capacidade de integração com os serviços locais de saúde, redefinindo qualitativamente seu modelo de atuação e mostrando que não é uma proposta de atenção simplificada e barata para áreas rurais e pobres do país.

A estratégia de Saúde da Família tem como pressuposto básico o trabalho em equipe, composta de vários profissionais de nível superior e médio, com o objetivo de realizar uma intervenção de caráter primordialmente preventivo e de promoção à saúde, em uma população territorialmente adscrita, visando especialmente à educação de grupos de risco, com maior propensão a adoecer ou a complicar, em função, muitas vezes, da falta de informação(2).

Neste modelo de assistência, a atenção está centrada na família entendida e percebida ejercicio físico, religión y cine. Entretanto, el centro de trabajo tiene reunión semanal programada con el equipo de salud para discusión de los problemas y sugerencias. Es indiscutible la importancia de intervenciones que promuevan resultados benéficos al trabajador a nivel psicológico y fisiológico, minimizando de esta manera, los efectos de los eventos generadores de estrés en el trabajo.

Palabras clave: Administración de la seguridad; Riesgos ocupacionales; Grupo de atención al Paciente; Salud de la Familia.

a partir do seu ambiente físico e social. Para assistir as famílias torna-se necessário a implantação de unidades de Saúde da Família que consistem em unidades ambulatoriais públicas de saúde destinadas a realizar assistência contínua nas especialidades básicas, por meio de equipe multiprofissional.

Essas equipes desenvolvem ações de promoção, prevenção, diagnóstico precoce, tratamento e reabilitação, características do nível primário de atenção, tendo como campos de intervenção o indivíduo, a família, o ambulatório, a comunidade e o meio ambiente. A unidade deve, portanto, estar vinculada à rede de serviços, de forma que se garanta atenção integral aos indivíduos e famílias, e sejam asseguradas a referência e contrareferência para clínicas e serviços de maior complexidade, sempre que o estado de saúde da pessoa exigir ${ }^{(3)}$

O profissional da Equipe de Saúde da Família precisa ser capaz de atuar com criatividade e senso crítico, mediante uma prática humanizada, competente e resolutiva.

Este modelo de assistência supõe que deva haver envolvimento dos trabalhadores das equipes com a população de seu território de abrangência, para o diagnóstico e intervenção na realidade sanitária.

No trabalho junto à comunidade é preciso integração e respeito, ouvindo os seus anseios e procurando buscar harmonia com a população a que se serve. Faz-se necessário que a equipe tenha maturidade e que haja desenvolvimento pessoal e profissional com enfrentamento de diversas realidades para que ocorram a 
promoção e a reabilitação da saúde destas famílias.

A experiência profissional na organização de Equipes de Saúde da Família nos mostrou que desenvolver este tipo de atividade junto à comunidade, onde a realidade do indivíduo fica muito próxima, os problemas são de diversas ordens, as limitações internas e externas incontáveis, expõem o trabalhador a riscos físicos e/ou psicossociais que podem desencadear o processo de estresse.

Camelo e Angerami(4), desenvolveram uma pesquisa na qual foram identificados riscos psicossociais no trabalho destes profissionais, tais como: falta de preparo e capacitação, sobrecarga de papéis, longas horas no trabalho, conflito no trabalho em equipe, dificuldade para conciliar trabalho e família e recursos materiais e humanos insuficientes. Estes riscos relacionam-se a aspectos de planejamento, organização e gerenciamento do trabalho, os quais apresentam potencial para causar prejuízo físico, social e psicológico podendo levar ao estresse $^{(5)}$.

O estresse tem sido considerado como um dos problemas que mais frequentemente age sobre 0 ser humano, interferindo na homeostase de seu organismo devido à grande quantidade de tensões que enfrenta diariamente.

A teoria do estresse fundamenta-se na avaliação de como o organismo responde às demandas do ambiente externo, sendo o estresse produzido em situações em que as demandas excedem as capacidades individuais de responder a esses estímulos. O modo como a pessoa lida com as circunstâncias geradoras de estresse exerce grande influência sobre sua saúde, modulando a gravidade do estresse resultante ${ }^{(6)}$.

Portanto, dentro do ambiente laboral é de suma importância que aprendamos a controlar o estresse de forma que ele venha a trazer benefícios individuais e grupais.

Em termos da presença de riscos ocupacionais, o gerenciamento destes envolve as decisões e ações que ocorrem em 2 níveis principais: 1) dentro da sociedade e pelos governos, através de políticas públicas, exigências legais, normas e padrões, que fundamentam a aceitabilidade de determinado risco e as práticas nos locais de trabalho; 2) no interior das empresas, através das práticas gerenciais que podem ajudar a prevenir os riscos nos locais de trabalho ${ }^{(7)}$.

Uma das formas conhecidas para aliviar o estresse no trabalho é a mudança organizacional permitindo, por exemplo, adequado tempo para o trabalhador realizar a sua tarefa, providenciando clara descrição de seu trabalho, permitindo autoridade no trabalho, promovendo tolerância e justiça no local de trabalho, eliminando exposições físicas prejudiciais no ambiente, identificando fracassos, sucessos, causas e conseqüências deles e, finalmente, melhorando o ambiente ocupacional e a saúde ${ }^{(8)}$.

Algumas estratégias podem ser desenvolvidas pelos trabalhadores, individualmente, no controle do estresse como(9): "Procurar ter saúde". Para a saúde, se requerem atitudes preventivas no sentido de se buscarem cuidados médicos constantemente. Especialmente em situações estressantes, uma quantidade maior de vitaminas será utilizada pelo organismo, havendo o desgaste e aumentando a probabilidade de doenças surgirem. Assim, uma alimentação saudável e balanceada é extremamente importante a fim de garantir a ingestão dos nutrientes necessários e evitar os excessos. "A prática de atividade física regular". Esta é uma estratégia de extrema importância no controle do estresse, favorecendo a produção no organismo de substâncias responsáveis pela sensação de bem-estar. "Saber relaxar". É uma atitude importante para manter o equilíbrio físico e mental, diminuindo estados de ansiedade e tensão.

O Programa de Saúde da Família é uma estratégia do Ministério da Saúde na qual se observa que são delegadas aos profissionais múltiplas tarefas com alto grau de exigências e responsabilidades. Estas, dependendo do ambiente e da organização do trabalho para a sua realização, e do preparo para exercer seu papel, podem levar o profissional a enfrentar um desequilíbrio biológico que afeta não só a ele como pessoa, mas também à comunidade assistida. Este desequilíbrio biológico, com componentes físicos e psicológicos conhecido como estresse, manifesta-se em forma de 
absenteísmo, alcoolismo, problemas emocionais, mudanças inexplicáveis na eficiência, desempenho irregular, impaciência com a população com quem lida e outros sérios problemas ${ }^{(10)}$.

A identificação de riscos psicossociais no trabalho das Equipes de Saúde da Família que podem levar ao estresse e as características organizacionais deste tipo de trabalho, pressupõe a necessidade de elaboração de estratégias para o controle destes riscos, e julgamos oportuno direcionar nossa pesquisa para estes aspectos.

Dessa forma, esta investigação tem o objetivo de identificar e analisar as estratégias utilizadas pelos trabalhadores para gerenciar os efeitos dos riscos psicossociais presentes no seu ambiente de trabalho.

\section{METODOLOGI A}

O trabalho desenvolvido é do tipo exploratório utilizando-se da abordagem qualitativa.

A unidade de campo foi constituída pelas unidades de saúde do município de Ribeirão Preto, que apresentava equipes de saúde da família mantidas exclusivamente pela Secretaria Municipal de Saúde e qualificadas pelo Sistema de Informação da Atenção Básica (SIAB) até janeiro de 2005 . Estas equipes iniciaram suas atividades a partir do ano de 2001 , e não houve rotatividade no quadro de pessoal até o momento da coleta de dados, que transcorreu de janeiro a março de 2005 .

Os sujeitos deste estudo são médicos, enfermeiros, auxiliares de enfermagem e agentes comunitários de saúde pertencentes a seis equipes de saúde da família, sendo um elemento de cada categoria profissional, escolhidos conforme disponibilidade para participação no ato da coleta de dados, perfazendo um total de 24 sujeitos.

Os dados foram coletados através de entrevistas semi-estruturadas as quais apresentaram informações referentes aos profissionais como: idade, sexo, estado civil e função exercida no local de trabalho. Também contou uma questão norteadora como : "Você utiliza algum recurso pessoal ou institucional para aliviar os efeitos de situações difíceis que enfrenta no dia-a-dia do seu trabalho?
A análise do presente estudo foi realizada através do método de análise de conteúdo de Bardin, modalidade temática(11). No conjunto de técnicas da análise de conteúdo, a análise por categorias é a mais utilizada e funciona por operações de desdobramento do texto em unidades, em categorias, segundo reagrupamentos analógicos. Entre as diferentes possibilidades de categorização, a análise temática é a mais eficaz. Fazer uma análise temática consiste em descobrir os núcleos de sentido que compõem a comunicação e cuja presença, ou freqüência de aparição pode trazer significados para o objetivo analítico escolhido ${ }^{(11)}$.

Nesse sentido, as discussões das unidades temáticas, depreendidas da análise do material coletado nas entrevistas, estão fundamentadas na identificação das estratégias de gerenciamento de riscos psicossociais relacionados ao trabalho das equipes de saúde da família segundo a percepção dos participantes desta investigação.

Esta pesquisa foi aprovada pelo Comitê de Ética em Pesquisa da Escola de Enfermagem de Ribeirão Preto-USP, protocolo $\mathrm{n} \times 0506 / 2004$. Os sujeitos, participantes desta investigação, assinaram o "Termo de Consentimento Livre e Esclarecido" de acordo com a Resolução do Conselho Nacional de Saúde no196/96.

\section{RESULTADOS E DISCUSSÃO}

Os resultados mostram que a equipe é predominantemente feminina $(87,5 \%)$, sendo mais evidente para os trabalhadores de enfermagem de nível médio e superior e agentes comunitários de saúde. Entre os trabalhadores de nível universitário, embora haja predomínio do sexo feminino, há presença masculina (50\%) entre os médicos. Esta situação se confirma em uma pesquisa que relata que o PSF absorve mais mulheres em suas equipes de trabalho(12).

Quando se olha para o perfil etário dos trabalhadores entrevistados, verifica-se que todos eles se encontram na faixa etária entre 35 e 56 anos, apontando para uma equipe que provavelmente teve oportunidade de vivenciar outras experiências profissionais e pessoais diversas, o que pode contribuir para um melhor 
desempenho no trabalho junto à Equipe de Saúde da Família.

Em relação ao estado civil, $16(66 \%)$ dos sujeitos são casados, cinco $(20,8 \%)$ solteiros e quatro $(12,5 \%)$ divorciados. Ao verificarmos que a maior parte dos trabalhadores de nossa pesquisa é casada, pode-se configurar um aumento do número de tarefas e responsabilidades. Este trabalhador deverá conciliar o trabalho e a família, de maneira que nenhuma das partes fique prejudicada.

\section{Estratégias de gerenciamento dos riscos no ambiente de trabalho}

A análise dos discursos identificou estratégias de gerenciamento dos riscos existentes no ambiente de trabalho, utilizadas pelo trabalhador, bem como, estratégias estabelecidas pela instituição, as quais estarão nas unidades temáticas que passaremos a descrever.

A partir da identificação destas estratégias foram construídas unidades temáticas relacionadas a elas, sendo: "Estratégias individuais" e "Estratégias de equipe".

\section{Estratégias individuais:}

Eu procuro ir andando pra casa, fazer caminhada, pra poder ir relaxando e voltar relaxada..." (ACS- Equipe 3 ).

$\mathrm{O}$ trabalho desenvolvido pelas Equipes de Saúde da Família envolve a presença de riscos psicossociais que podem levar ao estresse ${ }^{(4)}$. Estes riscos, presentes nas condições de trabalho, podem estar relacionados a cultura e função organizacional, o papel na organização, desenvolvimento na carreira, decisão e controle, relacionamento interpessoal no trabalho, interface trabalho-família, ambiente e equipamento de trabalho, planejamento de tarefas, cargas e local de trabalho e, finalmente, esquema de trabalho ${ }^{(5)}$

A identificação de estressores no ambiente laboral pressupõe a elaboração de estratégias para o seu controle a fim de evitar danos à saúde dos trabalhadores.

Existem estratégias para enfrentar os riscos no ambiente de trabalho que podem ser realizadas pelo trabalhador, independente da instituição, quais sejam: exercícios físicos, mudanças no estilo de vida, alimentação equilibrada, entre outras ${ }^{(13)}$

Os discursos revelam algumas destas estratégias utilizadas pelos trabalhadores para amenizar os efeitos desgastantes do seu trabalho.

Hoje, eu faço ginástica, tenho alguns cuidados, faço drenagem linfática uma vez por semana porque me faz bem... (MED- Equipe 4).

...Na minha vida pessoal, faço uma caminhada, nado um pouco. Em termos de lazer, eu vou ao cinema é isso... (MED- Equipe5).

Eu estava me desgastando! Eu não conseguia mais me aturar! É uma cobrança em cima da outra e você nunca está bem, você está sempre no regular... porque você malha, malha, malha e o paciente não faz. Então eu acho que isso desgasta e eu acabo ficando tensa. E eu fui buscar a saída em um curso de autoconhecimento, e eu melhorei muito... (ENF Equipe 2).

Os discursos revelam que as estratégias utilizadas pelos trabalhadores, como atividade física, repouso, terapia cognitivo comportamental, lazer, são de caráter pessoal, e indicam a busca do bem-estar emocional e atitudes adequadas para o enfrentamento desta realidade. Estas estratégias dependem única e exclusivamente do trabalhador para a sua realização.

Estudos mostram que geralmente há mais práticas de intervenção a nível individual para redução da exposição de riscos no ambiente de trabalho do que aquelas relacionadas a mudanças organizacionais ${ }^{(14)}$. A maioria dos programas de gerenciamento do estresse no trabalho focaliza técnicas como relaxamento e outras habilidades comportamentais, meditação, biofeedback e reestruturação cognitiva ou a combinação de algumas delas.

A carga horária, as longas horas de trabalho, o número de atividades desenvolvidas com a comunidade são riscos psicossociais percebidos pelos trabalhadores ${ }^{(4)} \mathrm{e}$ interferem decisivamente no tempo dedicado a si próprio, como aparece nos discursos dos entrevistados.

Eu faço hidroginástica, quando eu consigo assim adaptar meu horário direitinho eu faço academia, faço hidroginástica, quando eu não consigo fazer nada disso no horário certo eu 
faço caminhada, ouço música, leio, com os meus recursos (MED- Equipe 1).

Verificamos que o trabalho situa-se em primeiro plano na vida desses profissionais e somente quando a carga horária permite, é que eles vão em busca de estratégias para aliviar as tensões do ambiente laboral.

O exercício físico foi a estratégia mais abordada pelos trabalhadores de nossa pesquisa, $45 \%$ dos sujeitos da pesquisa. Segundo Weinberg e Gould ${ }^{(15)}$, as pesquisas apontam que indivíduos fisicamente ativos tendem a ter melhor saúde, demonstram energia, têm atitudes mais positivas em relação ao trabalho e revelam uma maior capacidade de lidar com o estresse e com a tensão.

A complexidade do Programa de Saúde da Família exige que os profissionais tenham clareza de suas diferentes dimensões: interesses e visões sobre os modelos de saúde, especialmente do PSF; visões sobre trabalho em equipe e compreensão da dinâmica das relações interpessoais entre equipe e comunidade. É preciso competência para intervir na trágica realidade sanitária brasileira, para que não assuma uma atitude acrítica e acomodada, pela insegurança em situar-se e atuar no Programa de Saúde da Família. A falta destes conhecimentos e habilidades deve provocar desgaste e insatisfação no trabalho.

Os trabalhadores de nosso estudo reconhecem a necessidade de aprimoramento técnico-científico a fim de lidar com as situações que se apresentam no cotidiano do trabalho, mas consideram que as estratégias utilizadas para aliviar as tensões favorecem esquecer os problemas e/ou dificuldades com os quais se deparam.

As entrevistas revelaram que os trabalhadores utilizam estratégias de gerenciamento ou controle das situações desgastantes após sofrerem o desgaste físico e/ou mental. Para alguns trabalhadores, a rotina intensa do trabalho faz com que ele procure uma ajuda somente quando se sente completamente desgastado.

Eu faço tricô... isso virou um hobby... agora quando eu estou estressada...eu faço pintura, artesanato queria aprender para ensinar o pessoal, mas acabou virando a minha terapia (ENF -Equipe 4).
Quando eu estou muito tensa, eu vou andar no supermercado ou ler... eu gosto muito de ler romance... às vezes eu estou tão cansada que eu não consigo fazer nada (AUX-Equipe 2).

As atividades desenvolvidas neste programa, não impedem os trabalhadores de procurarem descobrir novas formas de enfrentamento das situações adversas, como é o caso da religião.

Minha distração que alivia um pouco meu estresse são os cultos mesmo, a parte espiritual, a parte física não dá tempo (AUXEquipe 1).

A religião foi referenciada pelos auxiliares de enfermagem e agentes comunitários de saúde como uma estratégia para aliviar as tensões do trabalho. Ela deve propocionar a paz interior e ser o abrigo para o amparo espiritual de que as pessoas necessitam.

A religião pode melhorar a saúde promovendo práticas saudáveis de vida, melhorando o suporte social, oferecendo conforto em situações de estresse e sofrimento e até alterando substâncias químicas cerebrais que regulam o humor e a ansiedade. Portanto, ela parece ser um fator psicossocial benéfico na recuperação das doenças físicas e mentais ${ }^{(16)}$. Uma das funções das crenças religiosas pode ser a de alterarem a atividade do sistema imunológico, prevenindo dessa forma o estresse. Assim, pessoas religiosas teriam mais resistência aos fatores estressores do dia-a-dia, ou seja, melhor adaptação psicológica a elas ${ }^{(16)}$.

No momento em que os profissionais das Equipes de Saúde da Família fazem o máximo para atender às múltiplas exigências do trabalho altamente responsável, se deparando com situações desgastantes no dia-a-dia, enfrentam um desequilíbrio psicobiológico que pode vir a afetar não somente a eles como pessoa, mas também a comunidade assistida. Portanto, faz-se necessário que este trabalhador seja preparado para enfrentar esta realidade além de receber um suporte emocional adequado.

Um passo importante no gerenciamento das situações de risco é compreender nossas necessidades pessoais e profissionais em cada estágio de nossas vidas e reconhecer quando elas não estão sendo satisfeitas. 
Quando as pessoas estão perturbadas por algo que as está preocupando, isso as torna incapazes de operar adequadamente no trabalho.

Eu chego em casa e às vezes choro muito! Tem dia que eu nem como, porque tem coisa que você encontra... tem coisa que dá pra você tirar de letra, mas tem coisa que te emociona muito. Você encontra a pessoa numa situação muito difícil, aí é difícil para você. Aí eu acho que é só chorando mesmo para aliviar. A gente se apega muito nas pessoas.. (ACS- Equipe 2).

Esta fala retrata que, além do exercício físico, da leitura, religião, os trabalhadores também utilizam estratégias como o "choro" para aliviar as tensões do trabalho.

\section{Estratéqias da equipe:}

[...] Temos as nossas reuniões de equipe, onde a gente coloca as nossas alegrias, as nossas frustrações, as dificuldades que a gente está encontrando(ACS-Equipe 5).

De acordo com os discursos anteriores, podemos dizer que os trabalhadores encontram várias maneiras para aliviar as tensões do ambiente de trabalho. Mas não podemos esquecer que o local de trabalho pode ser uma fonte de ajuda ao trabalhador. A oferta de ajuda profissional é sempre bem-vinda.

Além das estratégias individuais, os trabalhadores utilizam estratégias coletivas como forma de aliviar as tensões do ambiente de trabalho, bem como melhorar o relacionamento entre os membros da equipe, que são as reuniões semanais de discussões de casos promovidas pela instituição.

Existem reuniões na instituição... essas reuniões, na realidade, elas são feitas pra gente tá buscando os nomes, os problemas, é mais assim uma troca entre as agentes comunitárias, equipe de enfermagem e a equipe do PSF da unidade. Então é uma troca de informação entre agente comunitária e a equipe do PSF, pra gente estar buscando essas informações e priorizando quais são as visitas mais importantes pra gente.. (AUX- Equipe 1).

A gente tem reunião semanal... aí a gente conversa de tudo, casos, agendar coisas, férias, informes, tudo... é uma reunião onde se fala de tudo. Se alguém está precisando desabafar alguma coisa é ali, é tudo ali. São umas duas horas de reunião (ENF - Equipe 5).

As reuniões aparecem em nossa pesquisa como uma estratégia comum a todas as equipes, onde há participação de todos os profissionais, com exposição das situações problemas e/ou necessidades da população, bem como, soluções para o cotidiano de seu trabalho. Geralmente estas reuniões são coordenadas por um membro da equipe, e realizadas em dia da semana pré-definido pela equipe ou gerente da unidade, mas existe a possibilidade de marcar encontros em outros períodos, de acordo com a necessidade.

Além desses encontros, não foi citado nos discursos dos trabalhadores outro tipo de estratégia oferecida pela instituição a que pertencem e observa-se nas suas falas a necessidade de um suporte para enfrentar as situações no coditiano do trabalho.

[...]A instituição não oferece nada, inclusive foi um pedido nosso. É muito difícil você trabalhar porque a gente tem agente comunitário de saúde que tem primeiro grau, tem agente que é mais...É muito difícil você lidar com esse grupo... Ás vezes o próprio grupo de agentes entra numas brigas entre eles, e pedimos uma assessoria, mas até agora não conseguimos (ENF- Equipe 1).

Através dos discursos, podemos observar que a falta de suporte institucional e a formação diferenciada dos membros da equipe gera desgaste para o grupo.

A menos que a organização tome uma posição clara com relação à questão do gerenciamento de situações estressantes, quaisquer ações individuais para tentar resolver o problema terão efeito limitado, particularmente dadas às inibições com relação a tratar do assunto ${ }^{(13)}$.

A instituição tem um papel fundamental no desenvolvimento de estratégias para o gerenciamento dos riscos do ambiente de trabalho.

Weimberg e Creed $^{(17)}$ propõem um enfoque baseado em evidências para melhorar o ambiente de trabalho que deveria ter dois componentes:

- um serviço de assistência individual ao funcionário, ou algum outro tratamento psicológico para 
assegurar a prevenção e o tratamento de doenças depressivas e

- $\quad$ suporte àquele que tem conflito entre suas funções clínicas e gerenciais fornecido pela organização.

Programas específicos de gerenciamento do estresse devem ser criados pelos serviços para cada situação. Os programas de manejo de estresse ocupacional podem ser focados na organização do trabalho e/ou no trabalhador. Intervenções focadas na organização são voltadas para a modificação de estressores do ambiente de trabalho, podendo incluir mudanças na estrutura organizacional, condições de trabalho, treinamento e desenvolvimento, participação e autonomia no trabalho e relações interpessoais no trabalho. Intervenções focadas no indivíduo almejam reduzir o impacto de riscos já existentes, através do desenvolvimento de um adequado repertório de estratégias de enfrentamento individuais ${ }^{(18)}$.

As organizações de trabalho devem ter o objetivo de restaurar o equilíbrio e funcionamento psicobiológico do trabalhador, para pelo menos amenizar problemas de estresse presentes.

\section{CONSI DERAÇÕES FINAIS}

O Programa de Saúde da Família é uma das estratégias recentes assumidas pelo Ministério da Saúde com vistas à reorganização da atenção básica à saúde. Os profissionais destas equipes assumem múltiplas tarefas com alto grau de exigência e de responsabilidades, para as quais, dependendo do ambiente da organização e planejamento do trabalho, da formação e preparo destes profissionais para exercer seu papel, expõem a riscos psicossociais que podem levar ao estresse.

A presença de riscos psicossociai,s relacionados ao trabalho de equipes de saúde da família, foram identificados e 0 seu gerenciamento faz-se necessário.

O controle dos riscos deve levar em consideração aspectos socio-econômicos, aspectos como a viabilidade tecnológica e a gestão adequada de recursos humanos, incorporando as melhores tecnologias disponíveis para a saúde dos trabalhadores.
O nosso estudo revela estratégias individuais utilizadas pelos profissionais para aliviar os efeitos das situações desgastantes às quais estão expostos, tais como: exercício físico, cinema, a música, a religião e o hábito da leitura. Observa-se também uma estratégia realizada pelo serviço, comum a todas as equipes, que são as reuniões semanais, onde há participação de todos os profissionais, com exposição das situações problemas e/ou necessidades da população, bem como, soluções para o cotidiano de seu trabalho.

No entendimento de que a realidade sanitária é dinâmica, pensamos que um serviço de apoio aos profissionais das Equipes de Saúde da Família deve, entre outras atividades, responder às necessidades individuais, orientando os trabalhadores sobre os riscos a que estão expostos, oferecendo suporte social e psicológico, regular encontros com profissionais habilitados, prover oportunidade para comunicar-se com outras equipes que têm experiência similar, promover e dar suporte para pesquisa. Programas específicos devem ser criados para cada situação.

Dessa forma, faz-se necessária adoção, pela referida instituição, de estratégias sadias de redução dos riscos do ambiente de trabalho voltadas para estes profissionais. É inquestionável a importância de elaboração de estratégias de promoção à saúde, ou seja, intervenções que promovam resultados benéficos ao trabalhador a nível psicológico e fisiológico, minimizando assim os efeitos dos eventos geradores de estresse no trabalho.

\section{REFERÊNCI AS}

1. Ministério da Saúde; Departamento de atenção básica. Guia Prático do Programa de Saúde da Família. Brasília (Brasil): Ministério da Saúde; 2001.

2. Santos MAM, Cutolo LRA. A interdisciplinaridade e o Trabalho em equipe no Programa de Saúde da Família. Arquivos Catarinenses de Medicina. 2003; 32(4): 65-74.

3. Santana ML, Carmagnani MI. Programa saúde da família no Brasil: um enfoque sobre os pressupostos básicos, operacionalização e vantagens. Saúde Sociedade. 2001;10(1):3353. 
4. Camelo SHH, Angerami ELS. Riscos psicossociais relacionados ao estresse no trabalho das Equipes de Saúde da Família: percepções dos profissionais. Rev Enferm UERJ. 2007; 15(4): 502-7.

5. Cox T, Rial-González E. Work-related stress: The European picture. Magazine of the European Agency for Safety and Health at work. 2002; (5): 4-6.

6. Beck CLC, Gonzáles RMB, Stekel LMC, Donaduzzi JC. O trabalho da enfermagem em unidades críticas e sua repercussão sobre a saúde dos trabalhadores. Esc Anna Nery. 2006; 10(2): 221-7.

7. Porto MFS. Análise de riscos nos locais de trabalho: conhecer para transformar. Cadernos de Saúde do Trabalhador. São Paulo: Kingraf; 2000.

8. European Commission. Guidance on workrelated stress- Spice of life or kiss of death? Luxembourg: European Communitites; 2000.

9. Nacarato AECB. Envelhecer é isto.... In: Lipp MEN, editor. O stress está dentro de você. São Paulo: Contexto; 1999. p.141-51.

10. Lipp MEN. Stress: conceitos básicos. In: Lipp MEN, editor. Pesquisas sobre stress no Brasil: Saúde, ocupações e grupos de risco. Campinas: Papirus; 1996. p. 17-31.

11. Bardin L. Análise de conteúdo. Lisboa: Edições 70; 1977.

12. Machado MH. Perfil dos médicos e enfermeiros do Programa de Saúde da Família no Brasil: relatório final. Brasília: Ministério da Saúde; 2000.

13. Warren E, Toll C. Como dominar seu stress: como indivíduos equipes e organizações podem equilibrar pressão e performance. Rio de Janeiro: Infobook; 1998.

14. European Agency for safety and health at work. Priorities for occupational safety and health research in the EU-25. Luxembourg: European Communities; 2005.

15. Weinberg RS, Gould D. Fundamento da Psicologia do esporte e do exercício. 2a edição. Porto Alegre: Editora Artmed; 2001.

16. Rennó Júnior J. A relação entre religião e medicina. Diário do Nordeste - CE [Internet]. 2005 maio 31; 2005 [cited 2008 dec 31]. Available from: http://sistemas.aids.gov.br/imprensa/Noticias.a sp?NOTCod $=64707$.
17. Weimberg A, Creed F. Stress and psychiatric disorder in healthcare professional and hospital staff. Lancet. 2000;2355(9203):533-7.

18. Calderero ARL, Miasso Al, Corradi-Webster CM. Estresse e estratégias de enfrentamento em uma equipe de enfermagem de Pronto Atendimento. Revista Eletrônica de Enfermagem [Internet]. 2008 [cited 2008 set 23]; 10(1):5162. Available from: http://www.fen.ufg.br/revista/v10/n1/v10n1a05.htm

Artigo recebido em 04.03.08.

Aprovado para publicação em 31.12.08. 